\title{
Neutrino Masses in Flipped SU(5)
}

\author{
G.K. Leontaris, and J.D. Vergados \\ Physics Department \\ University of Ioannina \\ Ioannina GR-451 10 \\ Greece
}

\begin{abstract}
$\underline{\text { ABSTRACT }}$
We analyse the fermion masses and mixings in the flipped SU(5) model. The fermion mass matrices are evolved from the GUT scale down to $m_{W}$ by solving the renormalization group equations for the Yukawa couplings. The constraints imposed by the charged fermion data are then utilised to make predictions about the neutrino properties . It is found that the generalized see-saw mechanism which occurs naturally in this model can provide $i$ )a solution to the solar neutrino problem via the MSW mechanism and ii)a sufficiently large $\nu_{\tau}$ mass to contribute as a hot dark matter component as indicated by the recent COBE data.
\end{abstract}

IOA-290

December 1992 
Nowadays there is a lot of experimental evidence that the neutrinos (or at least some of them) might have non-vanishing-although tiny-masses. Recent data from solar neutrino experiments $^{[1]}$ show that the deficiency of solar neutrino flux, i.e. the discrepancy between theoretical estimates and the experiment, is naturally explained if the $\nu_{e}$ neutrino oscillates to another species during its flight to the earth. In addition, new evidence has been reported ${ }^{[2,3]}$ for a significant depletion on the atmospheric $\nu_{\mu}$ flux. This can also be explained in terms of $\nu_{\mu} \longleftrightarrow \nu_{e}$ oscillations with mass difference of order $\Delta m^{2} \sim 10^{-2}-10^{-3} e V^{2}$ and a relatively large mixing angle $\left(\sin ^{2} 2 \theta_{\tau \mu} \geq .42\right)$. Furthermore, the COBE measurement [4] of the large scale microwave background anisotropy, might be explained ${ }^{[5]}$ if one assumes an admixture of COLD $(\sim 75 \%)$ plus HOT $(\sim 25 \%)$ dark-matter. It is hopefully expected that some neutrino (most likely $\nu_{\tau}$ ) may be the natural candidate of the hot dark matter component.

From the theoretical point of view, neutrino masses are zero in the minimal standard model of the electroweak intetractions. Non-zero neutrino masses arise naturally however, in most of the Grand Unified Theories (GUTs) as well as in Supersymmetric ones (SUSY GUTs). In all these models, neutrino masses are related to the quark masses. In general one usually obtains a Dirac type neutrino mass matrix very similar (or even identical) to the up-quark mass matrix at the GUT scale. Small neutrino masses in these models, compatible with the experimental constraints, are obtained in terms of the see-saw mechanism ${ }^{[6]}$. There, the left-handed $\nu$, and right-handed $\nu^{c}$ components of the neutrinos form the following mass matrix

$$
\left(\begin{array}{cc}
0 & m_{\nu_{D}} \\
m_{\nu_{D}}^{T} & M
\end{array}\right)
$$

where $m_{\nu_{D}}$ is the Dirac type $3 \times 3$ mass matrix $\left(\sim m_{u p}\right)$ while $\mathrm{M}$ is a $3 \times 3$ Majorana mass matrix with entries usually of the order of the GUT scale. After diagonalization one obtains small left-handed Majorana masses of the order of $m_{\nu} \sim m_{\nu_{D}}^{2} / M$ and heavy right-handed majorana states of order $M \sim M_{G U T}$. Light neutrino eigenmasses may then be evolved down to low energies and be compared with the experimental limits. The constraints put by the aforementioned neutrino data and their relation to the quark masses at the GUT scale is a real challenge for most of the proposed GUT models. Recently, motivated by the observed merging of the Standard Model gauge coupling constants in SUSY-GUTs there has been a revived interest in determining the low energy parameters of the theory in terms of few inputs at the GUT scale ${ }^{[7,8]}$ in the limit of zero neutrino masses. Since however most of the GUT models naturally predict the existence of right-handed neutrinos, the proposed framework has now been expanded ${ }^{[9,10]}$ to include non-vanishing neutrino masses as well. The general strategy in these approaches is to use the minimal number of parameters at the GUT scale so as to have the maximum number of predictions at $m_{W}$. Ultimately, one hopes that this minimal set of parameters at the GUT scale may be justified in terms of a more fundamental theory, such as the String Theory. We should point out, however, that not only $m_{\nu_{D}}$ is related to the up-quark masses but other indirect constraints come also from the rest of fermions. It thus appears challenging to utilize all possible such constraints in the mass matrix of eq.(1) in order to make definite predictions for the as yet elusive neutrinos which will then be checked by experiment, this way supporting or exluding such GUT scenarios. 
In the present work we would like to address the question of fermion and in particular the neutrino masses in GUT models which arise ${ }^{[11,12]}$ in the free fermionic construction of four dimensional strings. As an application we are going to consider the $S U(5) \times U(1)$ model of ref[11], but our results are valid also for the model of ref.[12]. There has been much fruitful work ${ }^{[13,14]}$ in this kind of models the last few years. Recently it was shown ${ }^{[15]}$ that the general see-saw mechanism which occurs naturally in this kind of models, turns out to be consistent with the recent solar neutrino data, while on the other hand suggests that CHOROUS and NOMAD experiments at CERN may have a good chance of observing $\nu_{\mu} \longleftrightarrow \nu_{\tau}$ oscillations. Here we are going to explore the neutrino masses in detail, assuming a specific ansatz for the quark and lepton mass matrices at the GUT scale, which is more or less dictated by some first attempts in deriving the above model from the four dimensional free fermionic Superstrings. As stated above, our general strategy is to use the minimum number of inputs so as to have the maximum number of predictions. We are going to make use of the GUT relations in order to fix these inputs in terms of well known low energy masses of the charged leptons as well as the $m_{u}$ and $m_{c}$ quarks and then to predict the rest of the fermion mass spectum.

The various tree-level superpotential mass terms which contribute to the neutrino mass matrix of the flipped $S U(5)$ model are the following:

$$
\lambda_{i j}^{u} F^{i} \bar{f}^{j} \bar{h}+\lambda_{i j}^{\phi \nu^{c}} F^{i} \bar{H} \phi^{j}+\lambda_{i j}^{\phi} \phi^{0} \phi^{i} \phi^{j}
$$

where in the above terms $F^{i}, \bar{f}^{j}$ are the $10, \overline{5}$ matter $\mathrm{SU}(5)$ fields while $\bar{H}, \bar{h}, h$ are the $\overline{10}, \overline{5}, 5$ Higgs representations and $\phi^{i}$ are neutral $S U(5) \times U(1)$ singlets. The Higgs field $\bar{H}$ gets a vacuum expectation value(v.e.v.) of the order of the SU(5) breaking scale $\left(\sim 10^{16} \mathrm{GeV}\right)$, $\bar{h}, h$ contain the standard higgs doublets while $\phi^{0}$ acquires a v.e.v. most preferably at the electoweak scale. The neutrino mass matrix may also receive significant contributions from other sources $^{[16],[13-15]}$. Of crusial importance, are the non-renormalizable contributions ${ }^{[16]}$ which may give a direct $M_{\nu^{c} \nu^{c}}=M^{\text {rad }}$ contribution which is absent in the tree-level potential. Then, the general $9 \times 9$ neutrino mass matrix in the basis $\left(\nu_{i}, \nu_{i}^{c}, \phi_{i}\right)$, may be written as follows:

$$
m_{\nu}=\left(\begin{array}{ccc}
0 & m_{U} & 0 \\
m_{U} & M^{r a d} & M_{\nu^{c}, \phi} \\
0 & M_{\nu^{c}, \phi} & \mu_{\phi}
\end{array}\right)
$$

where it is understood that all entries in eq.(3) represent $3 \times 3$ matrices. The above neutrino matrix is different from that of eq.(1), since now there are three neutral $S U(5) \times$ $U(1)$ singlets involved, one for each family. Note that this type of neutrino mass matrix with additional singlets, has also been introduced on purely phenomenological grounds, in various extensions of the standard model ${ }^{[17]}$. On the contrary, here the extended see-saw mechanism is natural in string derived models.

It is clear that the matrix (3) depends on a relatively large number of parameters and a reliable estimate of the light neutrino masses and the mixing angles is a rather complicated task. We are going to use however our knowledge of the rest of the fermion spectrum to reduce sufficiently the number of parameters involved. Firstly, due to the 
GUT relation $m_{U}\left(M_{G U T}\right)=m_{\nu_{D}}\left(M_{G U T}\right)$, we can deduce the form of $m_{\nu_{D}}\left(M_{G U T}\right)$, at the GUT scale in terms of the up-quark masses. The heavy majorana $3 \times 3$ matrix $M^{\mathrm{rad}}$, depends on the kind of the specific generating mechanism. For example, if $M^{\text {rad }}$ is due to some non-renormalizable interactions, then it is completely model dependent. Here,in order to be specific, motivated by the fact that in the non-supersymmetric version of the model this matrix may be generated radiatively ${ }^{[18]}$, we take it to be proportional to the down quark-matrix ${ }^{[14]}$ at the GUT scale:

$$
M^{r a d}=\Lambda^{r a d} m_{D}\left(M_{G U T}\right)
$$

The $M_{\nu^{c}, \phi}$ and $\mu_{\phi} 3 \times 3$ submatrices are also model dependent. In most of the string models however, there is only one entry at the trilinear superpotential in the matrix $M_{\nu^{c}, \phi}$, which is of the order $M_{G U T}$. Other terms, if any, usually arise from high order non-renormalizable terms. We will assume in this work only the existence of the trilinear term, since higher order ones will be comparable to $M^{\text {rad }}$ and are not going to change our predictions. In particular we will take $M_{\nu^{c}, \phi} \sim \operatorname{Diagonal}(M, 0,0)$, and $\mu_{\phi} \sim \operatorname{Diagonal}(\mu, 0,0)$, with $\mu<<M \sim M_{G U T}$, thus we will treat (3) as a $7 \times 7$ matrix.

Our ansatz for the other fermion mass matrices is

$$
\begin{gathered}
m_{U}=q\left(\begin{array}{lll}
0 & 0 & x \\
0 & y & z \\
x & z & 1
\end{array}\right) \equiv m_{\nu_{D}} \\
m_{D}=s\left(\begin{array}{lll}
0 & \alpha & 0 \\
\alpha & b & 0 \\
0 & 0 & f
\end{array}\right), m_{E}=s\left(\begin{array}{ccc}
0 & \alpha & 0 \\
\alpha & -3 b & 0 \\
0 & 0 & f
\end{array}\right)
\end{gathered}
$$

where

$$
s=\frac{v}{\sqrt{2}} \sin \beta, q=\lambda_{t}\left(t_{0}\right) \frac{v}{\sqrt{2}} \cos \beta
$$

and $\tan \beta=\frac{\bar{v}}{v} \equiv \frac{\bar{h}}{h}$. The form of the above mass matrices is considered at the GUT scale. In order to find their structure at the low energy scale and calculate the mass eigenstates as well as the mixing matrices and compare them with the experimental data, we need to evolve them down to $m_{W}$, using the renormalization group equations. Using the results of Ref.[19] we obtain the renormalization group equations for the Yukawa couplings at one-loop level

$$
\begin{gathered}
16 \pi^{2} \frac{d}{d t} \lambda_{U}=\left(I \cdot \operatorname{Tr}\left[3 \lambda_{U} \lambda_{U}^{\dagger}\right]+3 \lambda_{U} \lambda_{U}^{\dagger}+\lambda_{D} \lambda_{D}^{\dagger}-I \cdot G_{U}\right) \lambda_{U}, \\
16 \pi^{2} \frac{d}{d t} \lambda_{N}=\left(I \cdot \operatorname{Tr}\left[\lambda_{U} \lambda_{U}^{\dagger}\right]+\lambda_{E} \lambda_{E}^{\dagger}-I \cdot G_{N}\right) \lambda_{N} \\
16 \pi^{2} \frac{d}{d t} \lambda_{D}=\left(I \cdot\left(3 \operatorname{Tr}\left[\lambda_{D} \lambda_{D}^{\dagger}\right]+\operatorname{Tr}\left[\lambda_{E} \lambda_{E}^{\dagger}\right]\right)+3 \lambda_{D} \lambda_{D}^{\dagger}+\lambda_{U} \lambda_{U}^{\dagger}-I \cdot G_{D}\right) \lambda_{D},
\end{gathered}
$$




$$
16 \pi^{2} \frac{d}{d t} \lambda_{E}=\left(I \cdot\left(\operatorname{Tr}\left[\lambda_{E} \lambda_{E}^{\dagger}\right]+\operatorname{Tr}\left[\lambda_{D} \lambda_{D}^{\dagger}\right]\right)+3 \lambda_{E} \lambda_{E}^{\dagger}-I G_{E}\right) \lambda_{E}
$$

where $\lambda_{\alpha}, \alpha=U, N, D, E$, represent the $3 \times 3$ Yukawa matrices which are defined in terms of the mass matrices given in eqs. (4-6), and $\mathrm{I}$ is the $3 \times 3$ identity matrix. We have neglected one-loop corrections proportional to $\lambda_{N}^{2}$. $t \equiv \ln \left(\mu / \mu_{0}\right), \mu$ is the scale at which the couplings are to be determined and $\mu_{0}$ is the reference scale, in our case the GUT scale. The gauge contributions are given by

$$
\begin{gathered}
G_{\alpha}=\sum_{i=1}^{3} c_{\alpha}^{i} g_{i}^{2}(t), \\
g_{i}^{2}(t)=\frac{g_{i}^{2}\left(t_{0}\right)}{1-\frac{b_{i}}{8 \pi^{2}} g_{i}^{2}\left(t_{0}\right)\left(t-t_{0}\right)}
\end{gathered}
$$

The $g_{i}$ are the three gauge coupling constants of the Standard Model and $b_{i}$ are the corresponding beta functions in minimal supersymmetry. The coefficients $c_{i}^{\alpha}$ are given by

$$
\begin{gathered}
\left\{c_{U}^{i}\right\}_{i=1,2,3}=\left\{\frac{13}{15}, 3, \frac{16}{3}\right\},\left\{c_{D}^{i}\right\}_{i=1,2,3}=\left\{\frac{7}{15}, 3, \frac{16}{3}\right\}, \\
\left\{c_{E}^{i}\right\}_{i=1,2,3}=\left\{\frac{9}{5}, 3,0\right\},\left\{c_{N}^{i}\right\}_{i=1,2,3}=\left\{\frac{3}{5}, 3,0\right\},
\end{gathered}
$$

In the following, we find it convenient to redefine the quark and lepton fields such that $\lambda_{U}$ and $\lambda_{N}$ are diagonal

$$
\lambda_{U} \rightarrow \lambda_{U}=K^{\dagger} \lambda_{U} K, \lambda_{N} \rightarrow \lambda_{N}=K^{\dagger} \lambda_{N} K
$$

The matrix which diagonalizes the up quark mass matrix at the GUT scale is given by $(x<y<z)$

$$
K=\left(\begin{array}{ccc}
\frac{y-z^{2}}{D_{1}} & -\frac{x z}{D_{2}} & \frac{x y\left(1-y+z^{2}\right)}{\left(1+z^{2}\right) D_{3}} \\
\frac{x z}{D_{1}} & \frac{\left(y-z^{2}\right)}{D_{2}} & \frac{z y}{D_{3}} \\
-\frac{x y}{D_{1}} & -\frac{z\left(y-z^{2}\right)}{D_{2}} & \frac{y\left(1-y+z^{2}\right)}{D_{3}}
\end{array}\right)
$$

with

$$
\begin{aligned}
D_{1} & \approx\left(\left(y-z^{2}\right)^{2}\left(1+x^{2}\right)+x^{2} z^{2}\right)^{\frac{1}{2}} \\
D_{2} & \approx\left(\left(y-z^{2}\right)^{2}\left(1+z^{2}\right)+x^{2} z^{2}\right)^{\frac{1}{2}} \\
D_{3} & \approx\left(y^{2}\left(1-y+z^{2}\right)+y^{2} z^{2}\right)^{\frac{1}{2}}
\end{aligned}
$$

The mass eigenvalues at the GUT scale read:

$$
m_{1} \approx q \frac{-x^{2} y}{y-\left(x^{2}+z^{2}\right)}, m_{2}-m_{1} \approx q\left(y-\frac{x^{2}+z^{2}}{1-y}\right), m_{3} \approx q\left(1+\frac{x^{2}+z^{2}}{1-y}\right)
$$


We apply the field redefinitions (15) to the differential equations (7-10) and within the parentheses on the right hand side we retain only the dominant Yukawa coupling $\lambda_{t}^{2}(t)$

$$
\begin{gathered}
16 \pi^{2} \frac{d}{d t} \lambda_{U}=\left(\lambda_{t}^{2}(t)\left(\begin{array}{lll}
3 & & \\
& 3 & \\
& & 6
\end{array}\right)-G_{U}(t) I\right) \lambda_{U}, \\
\left.16 \pi^{2} \frac{d}{d t} \lambda_{N}=\left(\lambda_{t}^{2}(t)\left(\begin{array}{ccc}
1 & & \\
& 1 & \\
& & 1
\end{array}\right)-G_{N}(t) I\right)\right) \lambda_{N}, \\
\left.16 \pi^{2} \frac{d}{d t} \lambda_{D}=\lambda_{t}^{2}(t)\left(\begin{array}{ccc}
0 & & \\
& 0 & \\
& & 1
\end{array}\right)-G_{D}(t) I\right) \lambda_{D}, \\
16 \pi^{2} \frac{d}{d t} \lambda_{E}=-G_{E}(t) I \lambda_{E},
\end{gathered}
$$

Solving eqs.(18-21), we obtain:

$$
\lambda_{t}(t)=\lambda_{t}\left(t_{0}\right) \zeta^{6} \gamma_{U}(t)
$$

where

$$
\begin{gathered}
\gamma_{\alpha}(t)=\exp \left(-\int G_{\alpha}(t) d t /\left(16 \pi^{2}\right)\right) \\
=\Pi_{j=1}^{3}\left(\frac{\alpha_{j, 0}}{\alpha_{j}}\right)^{c_{\alpha}^{j} / 2 b_{j}} \\
=\Pi_{j=1}^{3}\left(1-\frac{b_{j, 0} \alpha_{j, 0}\left(t-t_{0}\right)}{2 \pi}\right)^{c_{\alpha}^{j} / 2 b_{j}} \\
\zeta=\exp \left(\frac{1}{16 \pi^{2}} \int_{t_{0}}^{t} \lambda_{t}^{2}(t) d t\right) \\
=\left(1-\frac{3}{4 \pi^{2}} \lambda_{\alpha}\left(t_{0}\right) \int_{t_{0}}^{t} \gamma_{t}^{2}(t) d t\right)^{-1 / 12}
\end{gathered}
$$

Then, the up quark masses are predicted to be:

$$
\begin{gathered}
m_{u} \approx \gamma_{U} \zeta^{3} q \frac{x^{2} y}{y-x^{2}-z^{2}} n_{u} \\
m_{c} \approx \gamma_{U} \zeta^{3} q\left(y-\frac{z^{2}+x^{2}}{1-y}\right) n_{c}+\frac{\eta_{c}}{\eta_{u}} m_{u}
\end{gathered}
$$




$$
m_{t} \approx \gamma_{U} \zeta^{6} q\left(1+\frac{x^{2}+z^{2}}{1-y}\right)
$$

In the above formulae, $\eta_{u}$ and $\eta_{c}$ are taking into account the effects of QCD renormalization from the scale $m_{t}$ down to $1 G e V$ for $m_{u}$ and to $m_{c}$ for $m_{c}$.

Similarly, renormalizing $\lambda_{N}$ down to $m_{t}$ and expressing the eigenvalues in terms of the up-quark masses, we find that the Dirac-neutrino masses are

$$
m_{\nu_{D_{1}}} \approx \frac{\gamma_{N}}{\gamma_{U}} \frac{1}{\eta_{u} \zeta^{2}} m_{u}, m_{\nu_{D_{2}}} \approx \frac{\gamma_{N}}{\gamma_{U}} \frac{1}{\eta_{u} \zeta^{2}} m_{c}, m_{\nu_{D_{3}}} \approx \frac{\gamma_{N}}{\gamma_{U}} \frac{1}{\zeta^{5}} m_{t}
$$

In the above basis where the up quark and neutrino matrices are diagonal, the renormalized down quark mass matrix is found to be

$$
m_{D}^{r e n} \approx \gamma_{D}\left(\begin{array}{ccc}
1 & & \\
& 1 & \\
& & \zeta
\end{array}\right) s K^{\dagger}\left(\begin{array}{ccc}
0 & \alpha & 0 \\
\alpha & b & 0 \\
0 & 0 & f
\end{array}\right) K
$$

while for the leptons one gets the matrix

$$
m_{E}^{r e n} \approx \gamma_{E} s K^{\dagger}\left(\begin{array}{ccc}
0 & \alpha & 0 \\
\alpha & -3 b & 0 \\
0 & 0 & f
\end{array}\right) K
$$

We consider the lepton masses as inputs, and we find the approximate expressions for the down quarks in terms of the leptons to be:

$$
\begin{gathered}
m_{b} \approx \frac{\gamma_{D}}{\gamma_{E}} \zeta m_{\tau} n_{b} \\
m_{d} \approx-n_{d} \frac{\gamma_{D}}{6 \gamma_{E}}\left(m_{\mu}-m_{e}-\sqrt{\left(m_{\mu}-m_{e}\right)^{2}+36 m_{\mu} m_{e}}\right) \\
m_{s} \approx n_{s} \frac{\gamma_{D}}{6 \gamma_{E}}\left(m_{\mu}-m_{e}+\sqrt{\left.\left(m_{\mu}-m_{e}\right)^{2}+36 m_{\mu} m_{e}\right)}\right)
\end{gathered}
$$

where now, $\eta_{d}, \eta_{s}$ and $\eta_{b}$, are taking into account the QCD renormalization effects for the corresponding down quarks and $\zeta, \gamma_{\alpha}$ are given in terms of (23)-(27). We will take $\eta_{d, s, u} \approx 2, \eta_{c} \approx 1.8$ and $\eta_{b} \approx 1.4$ Now, since the range of the charged lepton masses are well known, one can use the above equations to determine the corresponding range of the down quarks and compare it with the running masses of $d, s$ and $b$. The range of the latter, is determined via SU(4) mass relations or QCD sum rules ${ }^{[20]}$. Thus, for example, from $\mathrm{SU}(4)$ mass relations one gets

$$
m_{d}=7.9 \pm 2.4 M e V, m_{s}=155 \pm 50 M e V,
$$

and from QCD sum rules

$$
m_{d}=8.9 \pm 2.6 \mathrm{MeV}, m_{s}=175 \pm 55 \mathrm{MeV},
$$


while $m_{b}=4.25 \pm .10 \mathrm{GeV}$. An interesting fact is that the renormalization parameter $\zeta$ is constrained in a narrow region in order to give the correct prediction for the bottom mass. For all the acceptable $m_{t}$ range $\frac{\gamma_{D}}{\gamma_{E}} \approx 2.1$ and $\eta_{b} \approx 1.4$, thus $\zeta \approx .81 \pm .2$. The predictions of the other two down quark masses are $m_{s} \approx 153 \mathrm{MeV}$ and $m_{d} \approx 6.3 \mathrm{MeV}$. The $m_{s}$ value is within the acceptable ranges given in (37-38). $m_{d}$ value is somewhat low but still in the range of (37).

The Kobayashi-Maskawa (KM) matrix can be determined by diagonalizing the downquark matrix in (32). In order to determine the KM-mixing angles, we first determine the values of the parameters of $x, y, z$ which give the correct masses $m_{u}=5.1 \pm 1.5 \mathrm{MeV}, m_{c}=$ $1.27 \pm .05 \mathrm{GeV}$, while always we adjust properly $\tan \beta$ and $\lambda_{t}\left(t_{0}\right)$, so as to obtain the correct value for $m_{b}$. It is worth noting here that the restricted region of $\zeta$ has a significant impact on the $m_{t}$ value. Indeed, as $m_{t}$ gets smaller, the range $\left(M_{G U T}-m_{t}\right)$ becomes bigger, thus the value of $\zeta$ increases. For $M_{G U T} \approx 10^{16} \mathrm{GeV}$, (using $\eta_{b} \approx 1.4$ ), we find that $m_{b}$ is pushed to its upper limit, when $m_{t}$ is around $125 \mathrm{GeV} . m_{b}$ goes to its lower limit as $m_{t}$ approaches $175 \mathrm{GeV}$, while $m_{u}\left(m_{c}\right)$ gets its lower(higher)acceptable value. We also keep track of the ratios $15 \leq \frac{m_{s}}{m_{d}} \leq 25, .2 \leq \frac{m_{u}}{m_{d}} \leq .7$, which are constrained by chiral Lagrangian analyses $^{[21]}$. Here, they are found $\approx 24.5$ and $\approx .65$ respectively. Proceeding further, we determine numerically the KM-matrix for each case seperately. Then, we return to the neutrino mass matrix and find the mass eigenstates as well as the diagonalizing matrix. Then, if $S^{\nu}$ is the matrix which diagonalizes the effective $3 \times 3$ light neutrino sector and $S_{e}^{L}$ the charged lepton mixing matrix, the leptonic mixing matrix is defined as follows:

$$
V^{l e p}=S^{\nu} S_{e}^{L \dagger}
$$

In the following we present numerical results for some characteristic values of the $m_{t}$ mass. We start running the R.G.E.s from the scale $M_{G U T} \approx 10^{16} \mathrm{GeV}$ (which is known to be the scale where the standard model gauge couplings meet ${ }^{[22]}$, while the value for the common gauge coupling at $M_{G U T}$ is taken $g_{G U T}=\frac{1}{25.1}$. We will assume that supersymmetry is valid down to the scale $m_{t}$ while we run the system with the nonsupersymmetric beta function coefficients bellow $m_{t}$. First we determine the quark and charged lepton masses, mixings etc which are described in terms of 13 free parameters in the context of the standard model, only with the eight input parameters $(x, y, z, q, \phi, a, b, f)$ at the GUT scale. Using only two additional inputs which are the scales of the $\nu^{c} \nu^{c}$ and $\nu^{c} \phi$ entries in the neutrino mass matrix, we give predictions for the light neutrino masses and leptonic mixing angles which can be tested in recent neutrino experiments. Taking into account all the constraints and mass relations mentioned above, we present in the following our results for $m_{t}=130,150$ and $160 \mathrm{GeV}$. We always choose to fix $a, b$ and $f$ parameters in terms of the charged lepton masses, hence we give our results only in terms of the set $(x, y, z, \phi)$ and $\tan \beta$. Then, $\lambda_{t}\left(t_{0}\right)$ coupling is also fixed once $\tan \beta$ and $m_{t}$ are chosen.

For $m_{t}=130 G e V, \tan \beta=1.1$ and $\phi=\frac{\pi}{6.5}$, we obtain the following results:

$$
\begin{array}{r}
m_{d} \approx 6.3 \mathrm{MeV}, m_{s} \approx 154 \mathrm{MeV}, m_{b} \approx 4.33 \mathrm{GeV} \\
m_{u} \approx 4.0 \mathrm{MeV}, m_{c} \approx 1.27 \mathrm{GeV}
\end{array}
$$


in agreement with the values obtained by the approximation formulae (28-30) and (34-36). The Kobayashi-Maskawa matrix elements $\left|\left(V_{K M}\right)_{i j}\right|$, are

$$
V_{K M}=\left(\begin{array}{lll}
.9754 & .2205 & .0032 \\
.2202 & .9748 & .0356 \\
.0108 & .0340 & .9994
\end{array}\right)
$$

For $m_{t}=150 \mathrm{GeV}$, we use $\tan \beta=2.2$ and $\phi=\frac{\pi}{4.5}$. We get

$$
\begin{array}{r}
m_{d} \approx 6.2 \mathrm{MeV}, m_{s} \approx 153 \mathrm{MeV}, m_{b} \approx 4.25 \mathrm{GeV} ; \\
m_{u} \approx 4.05 \mathrm{MeV}, m_{c} \approx 1.26 \mathrm{GeV} \\
V_{K M}=\left(\begin{array}{lll}
.9752 & .2212 & .0028 \\
.2109 & .9744 & .0429 \\
.0117 & .0413 & .9991
\end{array}\right)
\end{array}
$$

Finally, for $m_{t}=160 \mathrm{GeV}$, we take $\tan \beta \approx 3.3$ and $\phi=\frac{\pi}{4.5}$.Then,

$$
\begin{array}{r}
m_{d} \approx 6.2 \mathrm{MeV}, m_{s} \approx 152 \mathrm{MeV}, m_{b} \approx 4.26 \mathrm{GeV} ; \\
m_{u} \approx 3.9 \mathrm{MeV}, m_{c} \approx 1.26 \mathrm{GeV} \\
V_{K M}=\left(\begin{array}{lll}
.9751 & .2219 & .0025 \\
.2216 & .9741 & .0445 \\
.0120 & .0430 & .9990
\end{array}\right)
\end{array}
$$

It is worth noting here, that as the top mass gets higher the phase $\phi$ should also become larger in order for the KM-entries to lie within the experimental limits. A larger $\tan \beta$ is also required.

To obtain the neutrino spectrum and lepton mixing, we must introduce values for the two additional papameters $M, \Lambda^{r a d}$ of the neutrino mass matrix (3). We assume naturally $M=<\bar{H}>\approx 10^{16} \mathrm{GeV}$. In order to study the properties of the neutrino matrix, we let $\Lambda^{\text {rad }}$ vary in a reasonable range between $10^{11}$ and $10^{13}$ and fix its value later with the available neutrino data.

Next, we parametrize the lepton mixing matrix in a convenient way,i.e.

$$
V^{l e p}=\left(\begin{array}{ccc}
c_{1} c_{3}-s_{1} s_{2} s_{3} e^{i \phi} & s_{1} c_{3}+c_{1} s_{2} s_{3} e^{i \phi} & -c_{2} s_{3} \\
-s_{1} c_{2} e^{i \phi} & c_{1} c_{2} e^{i \phi} & s_{2} \\
c_{1} s_{3}+s_{1} s_{2} c_{3} e^{i \phi} & s_{1} s_{3}-c_{1} s_{2} c_{3} e^{i \phi} & c_{2} c_{3}
\end{array}\right)
$$

The predictions of the relevant mixing for the neutrino oscillations can now be presented in terms of the angles defined in the parametrization of $V^{l e p}$.

In our model described above $V^{l e p}$ is fixed by the quark and charged lepton data. In fact, due to the assumed form of the matrix $M_{\nu^{c}, \phi}$, it only depends on the ratio $\frac{M_{33}^{\text {rad }}}{M_{11}^{\text {rad }}}$ which in our model is equal to $\frac{b}{f}$ (see equs (4) and (6a)). The neutrino eigenvalues, however, 
cannot be accurately predicted due to the scale quantity $\Lambda^{\text {rad }}$ which is not specified in our model. Thus they can be written as

$$
m_{\nu_{e}} \approx 0, m_{\nu_{\mu}}=\frac{\Lambda^{\mu}}{\Lambda^{r a d}} \times 10^{-2} e V, m_{\nu_{\tau}}=\frac{\Lambda^{\tau}}{\Lambda^{r a d}} \times 10 \mathrm{eV}
$$

For $m_{t} \approx 130 \mathrm{GeV}$ we get $\Lambda^{\mu} \approx .80 \times 10^{12}$ and $\Lambda^{\tau} \approx 1.85 \times 10^{12}$.

Next, we present the light neutrino mixing matrices for two choices of $m_{t}$, i.e. $m_{t}=$ $130 \mathrm{GeV}$ and $m_{t}=150 \mathrm{GeV}$.

For $m_{t}=130 \mathrm{GeV}$, we obtain

$$
V^{l e p}=\left(\begin{array}{ccc}
-.9958+7.7 \imath \times 10^{-4} & (-8.5+3.2 \imath) \times 10^{-2} & .00347 \\
(-8.5-3.2 \imath) \times 10^{-2} & .9954+7.7 \imath \times 10^{-4} & -.0307 \\
(-8.4+9.9 \imath) \times 10^{-4} & -.031+.09 \imath \times 10^{-3} & -.9995
\end{array}\right)
$$

For $m_{t}=150 \mathrm{GeV}$ we get

$$
V^{l e p}=\left(\begin{array}{ccc}
-.9955+1.4 \imath \times 10^{-3} & (8.4-4.5 \imath) \times 10^{-2} & .0034 \\
(-8.4-4.5 \imath) \times 10^{-2} & -.9947-1.37 \imath \times 10^{-3} & -.0388 \\
(-.13+1.7 \imath) \times 10^{-3} & .039-.098 \imath \times 10^{-3} & -.9993
\end{array}\right)
$$

From the above, it can be seen that the mixing between all neutrino species is small. For values of $\Lambda^{\text {rad }} \approx 10^{12}$ the obtained neutrino masses are much too small to be detected directly in present experiments like neutrinoless double beta decay, muon number violating processes etc. At present, the only place to detect such small neutrino masses are neutrino oscillation experiments or astrophysics. We can approximate the oscillation probabilities relevant to this latter case with a high accuracy in terms of the $2 \times 2$ familiar case, as follows

$$
\begin{aligned}
& P\left(\nu_{e} \leftrightarrow \nu_{\mu}\right) \approx 3.1 \times 10^{-2} \sin ^{2}\left(\pi \frac{L}{l_{12}}\right) \\
& P\left(\nu_{\tau} \rightarrow \nu_{\mu}\right) \approx 4.0 \times 10^{-3} \sin ^{2}\left(\pi \frac{L}{l_{13}}\right) \\
& P\left(\nu_{e} \rightarrow \nu_{\tau}\right) \approx 4.0 \times 10^{-5} \sin ^{2}\left(\pi \frac{L}{l_{13}}\right)
\end{aligned}
$$

where $\mathrm{L}$ is the source-detector distance and

$$
l_{i j}=\frac{4 \pi E_{\nu}}{\left|m_{i}^{2}-m_{j}^{2}\right|}
$$

Notice that the oscillation length $l_{23}$ does not appear in the above formulae. Since however, $m_{\nu_{e}} \ll m_{\nu_{\mu}}$ and $m_{\nu_{\mu}} \ll m_{\nu_{\tau}}$, one can in principle constrain both $m_{\nu_{\mu}}$ and $m_{\nu_{\tau}}$ from such data. It is clear from the relations (45a-45c) that our results are not compatible with large mixing angle experimental limits. Neutrino oscillations in the medium ${ }^{[23]}$ via the MSW 
effect $^{[24]}$ provide a solution to the solar neutrino problem. The GALLEX solar neutrino data $^{[25]}$

$$
\begin{gathered}
5.0 \times 10^{-3} \leq \sin ^{2} 2 \theta_{i j} \leq 1.6 \times 10^{-2} \\
0.32 \times 10^{-5}(\mathrm{eV})^{2} \leq \delta m_{i j}^{2} \leq 1.2 \times 10^{-5}(\mathrm{eV})^{2}
\end{gathered}
$$

can be accomodated in our model. Our result (45a) is a bit outside the above range but the mass constraint can be easily satisfied by choosing $\Lambda^{\text {rad }}$ in the range

$$
.7 \times 10^{12} \leq \Lambda^{r a d} \leq 7 \times 10^{12}
$$

Our neutrino masses can also easily be made to fall into the range of the Frejus atmospheric neutrinos ${ }^{[26]}$

$$
10^{-3}(e V)^{2} \leq \delta m_{i j}^{2} \leq 10^{-2}(e V)^{2}
$$

but our mixing is much too small. Our results are also consistent with the data on $\nu_{\mu} \leftrightarrow \nu_{\tau}$ oscillations $^{[26]}$

$$
\sin ^{2} 2 \theta_{\mu \tau} \leq 4 . \times 10^{-3}, \delta m_{\nu_{\mu} \nu_{\tau}}^{2} \geq 50(e V)^{2}
$$

Our results however cannot be made to fall on the $\sin ^{2} 2 \theta$ vs $\delta m^{2}$ of the $B N L \nu_{\mu} \leftrightarrow \nu_{e}$ oscillation results ${ }^{[27]}$.

Moreover, it is always possible to obtain $m_{\nu_{\tau}} \approx($ few $\sim 20) \mathrm{eV}$, hence one can obtain simultaneously the cosmological HOT-dark matter component, in agreement with the interpretation of the COBE data ${ }^{[28]}$. Indeed an upper limit on the $\nu_{\tau}$ mass can be obtained from the formula

$$
7.5 \times 10^{-2} \leq \Omega_{\nu} h^{2} \leq 0.3
$$

Translating this into a constraint on $m_{\nu_{\tau}}$, arising from the relation $m_{\nu_{\tau}} \approx \Omega_{\nu} h^{2} 91.5 \mathrm{eV}$ where $h=.5 \sim 1.0$ is the Hubble parameter, one gets the range

$$
6.8 \leq m_{\nu_{\tau}} \leq 27 e V
$$

which can be easily achieved with the above range of $\Lambda^{\text {rad }}$.

In conclusion, we have proposed a structure of the fermion mass matrices in the flipped $\mathrm{SU}(5)$ model. By allowing the Yukawa couplings to evolve from the GUT scale down to $m_{W}$, using only 8 input parameters at the GUT scale, we can fix all the 13 measurable parameters (masses and mixings angles), at $m_{W}$. Furthermore, with the above information our model allows us to make definite predictions for the neutrino masses and the leptonic "Kobayashi Maskawa" matrix. In particular, we have found that the generalized see-saw mechanism which occurs naturally in this model can provide a solution to the solar neutrino problem via the MSW mechanism. Moreover, a sufficiently large $\nu_{\tau}$ mass is always possible in this model in order to contribute as a hot dark matter component, as indicated by the recent $\mathrm{COBE}$ data.

Note added. After the completion of this work we received a copy of the paper of ref[29], where it is shown that the generalized see-saw mechanism in this model can also account for the baryon asymmetry of the Universe. Indeed, in our model we also have at least one singlet neutrino state with mass of order $10^{11} \mathrm{GeV}$. 


\section{Acknowledgements}

We would like to acknowledge partial support by the EC-grant SCI-0221-C(TT) 


\section{$\underline{\text { REFERENCES }}$}

[1] K.S. Hirata et al Phys. Rev Lett. 65(90) 1297;

K.S. Hirata et al, Phys. Rev. D44 (91) 2141 ;

SAGE collaboration A.I.Abazov et al, Phys.Rev.Lett.67(91) 3332;

[2] K.S. Hirata, et al, Kamiokande-II Collaboration (Phys.Lett.B) ;

[3] R Becker-Szendy et al Boston Univ. preprint BUHEP-91-2491992)

[4] G.F. Smooth et al,Astrophys.J. Lett. (to appear).

[5] JR.K. Schaefer and Q.Shafi Ic/92/118 (BA-92-45);

[6] T.Yanagida, Prog. Th. Phys. B 135 (1978) 66 ;

M. Gell Mann P. Ramond and Slansky, ;

in Supergravity,ed P.van Niewenhuizen and D. Freedman ;

(North Holland 1979 p.315);

R. Mohapatra and G. Senjanovic, Phys.Rev.Lett.44(1980)912.

[7] S. Dimopoulos, L. J. Hall and S. Raby,

Phys. Rev. Lett.68(92) 1984; Phys. Rev D 45 (92) 4192;

V. Barger et al Phys. Rev. Lett 68 (1992)3394.

[8] G. F. Giudice, UTTG-5-92, March 1992, Texas Univ. Preprint;

R.G. Roberts and G.G. Ross, Oxford Preprint (92);

K.S.Babu and Q. Shafi, Univ. of Delaware preprint.

[9] S.Dimopoulos, L.J. Hall and S. Raby LBL-32484/92,August.

[10] H. Dreiner, G.K. Leontaris and N.D. Tracas, Oxford;

preprint,September 1992;IOA-281/92

[11] I. Antoniadis et al, Phys. Lett. B 194 (1987) 231;

[12] I. Antoniadis and G.K. Leontaris Phys. Lett. B 216(1989) 333;

[13] G.K. Leontaris Phys. Lett. B 207(1988)447;

G.K. Leontaris and D.V. Nanopoulos Phys. Lett. B 212(1988)327;

G.K. Leontaris and C.E. Vayonakis, Phys. Lett. B206(1988)271;

S. Abel, Phys.Lett.B234(1990)113;

I. Antoniadis, J.Rizos and K.Tamvakis Phys.Lett.B279(1992)281;

[14] G.K. Leontaris and J.D.Vergados Phys. Lett. B258(1991)111;

E. Papageorgiu and S. Ranfone Phys.Lett.B282(1992)89;

[15] J. Ellis, J.L. Lopez and D.V. Nanopoulos Phys.Lett.B(1992);

[16] S.Kalara, J.L. Lopez and D.V. Nanopoulos, Phys.Lett.B245(1990)421;

[17] J.W.F. Valle," Gauge theories and the physics of neutrino mass;

Progress in Part. and Nucl. Phys. Vol.26,1991.

[18] E.Witten, Phys.Lett.B91(1980)81; 
[19] R. Barbieri et al., Phys.Lett.B155(1982)212;

M.B.Einhorn and D.R.T.Jones, Nucl.Phys.B196(1982)457;

J.P.Derendinger and C.A. Savoy, Nucl.Phys.B237(1984)307;

B.Gato et al., Nucl.Phys.B253(1985)285;

N.K. Falck, Zeit. Phys. C 30(1986) 247.

[20] J.Gasser and H. Leutwyler, Phys.Rep.87(1982)77

[21] D. Kaplan and A. Manohar, Phys.Rev.Lett.56(1986)2004;

H. Leutwyler, Nucl.Phys.B337(1990)108.

[22] J. Ellis, S. Kelley and D.V. Nanopoulos, Phys. Lett. B 249 (1990)441;

P. Langacker and M. Luo, Phys. Rev. D 441 (1991) 817;

U.Amaldi, W. de Boer, and H. Fürstenau, Phys. Lett. B 260 (1991) 447;

R.G. Roberts and G.G. Ross, Nucl. Phys. B 377 (1992) 571.

[23] P.I. Krastev and S.T. Petcov,CERN-TH 6539/92;

S.M. Bilenky, Neutrino mixing DFTT67/92.

[24] L. Wolfenstein, Phys.Rev.D17(1978)2369;

S.P. Mikheyev and A.Yu. Smirnov, Yar.Fiz.42(1985)1441.

[25] P. Anselmann et al. Phys.Lett.285(1992)376

[26] Ch. Berger et al Phys. Lett.B245(1990)305

[27] L. Borodovsky et al, Phys.Rev.Lett.68(1992)247

[28] F.L. Wright et al. Ap.J.Lett.396(1992)L13

[29] J. Ellis, D.V. Nanopoulos and K.A. Olive, "Flipped heavy neutrinos: from the solar neutrino problem to baryogenesis" CERN-TH6271/92,ACT-22/92,UMN-TH1117/92 


\section{TABLE 1.}

\section{$\mathrm{m}_{\mathrm{t}}=130 \mathrm{GeV}$}
$\Lambda_{\text {rad }}$
$0.50 \times 10^{12}$
$0.75 \times 10^{12}$
$1.00 \times 10^{12}$
$3.00 \times 10^{12}$
$5.00 \times 10^{12}$
$7.00 \times 10^{12}$

$\mathbf{m}_{\mu}$

$\mathbf{m}_{\tau}$

$1.61 \times 10^{-2}$

$36.9 \mathrm{eV}$

$1.08 \times 10^{-2}$

$24.6 \mathrm{eV}$

$0.80 \times 10^{-2}$

$18.5 \mathrm{eV}$

$0.27 \times 10^{-2}$

$6.2 \mathrm{eV}$

$0.16 \times 10^{-2}$

$3.7 \mathrm{eV}$

$0.12 \times 10^{-2}$

$2.6 \mathrm{eV}$

\section{$\mathrm{m}_{\mathrm{t}}=150 \mathrm{GeV}$}

$\begin{array}{lll}\mathbf{V}_{\mathbf{e} \mu}^{\mathbf{l e p}} & \mathbf{V}_{\mu \tau}^{\mathbf{l e p}} & \mathbf{V}_{\tau \mathbf{e}}^{\mathbf{l e p}} \\ (8.38-\imath 4.46) \times 10^{-2} & 3.88 \times 10^{-2} & (-.125+\imath 1.74) \times 10^{-3} \\ \boldsymbol{\Lambda}_{\mathbf{r a d}} & \mathbf{m}_{\mu} & \mathbf{m}_{\tau} \\ 0.50 \times 10^{12} & 1.68 \times 10^{-2} & 59.7 \mathrm{eV} \\ 0.75 \times 10^{12} & 1.12 \times 10^{-2} & 39.8 \mathrm{eV} \\ 2.00 \times 10^{12} & 0.42 \times 10^{-2} & 14.9 \mathrm{eV} \\ 5.00 \times 10^{12} & 0.17 \times 10^{-2} & 5.9 \mathrm{eV} \\ 7.00 \times 10^{12} & 0.12 \times 10^{-2} & 4.3 \mathrm{eV}\end{array}$

